\title{
Electronic Transport through a Bending Contact
}

\author{
U. SCHRÖTER \\ FB Physik, AG Scheer, Universität Konstanz \\ Universitätsstraße 10, 78457 Konstanz, Germany
}

\begin{abstract}
The so-called "break-junction technique" allows us to realize contacts only single-atom in diameter at their thinnest point. Electronic transport through such narrow constrictions shows a quantum mechanical signature which is well described in a transport channel picture. Each individual contact is characterized by an ensemble of channel transmission probabilities. Preferred total conductance values as well as the number of channels, however, turn out to be universal for the material investigated, despite the lack of control of the exact contact geometry.
\end{abstract}

PACS numbers: 73.23.-b, 74.50.+r, 74.45.+c

\section{Introduction}

The diameter of a connection between two conducting reservoirs is in principle limited to the size of an atom. Different methods have been pursued to fabricate contacts with a cross-section of a single or a few atoms or at least sufficiently small such that the wave nature of the electrons makes transport through the contact governed by quantum mechanics [1]. By electron-beam lithography and reactive ion etching it is possible to form holes in a silicon-nitride sheet, the contact through which is then established by metal evaporation from both sides [2]. With this method the contact area is fixed for each sample and cannot go below several nanometers in diameter. An aluminum stripe connecting two metallic reservoirs can be further and further reduced in width and height when oxidizing it by scanning an atomic force microscope (AFM) tip across it to which a voltage is applied relative to the substrate [3]. In contrast to such lithographic methods a conducting connection can be assembled - and also be dissolved again — atom by atom between a scanning tunneling microscope (STM) tip and a substrate in an electrolyte solution $[4,5]$ by electrochemical deposition. Mechanical ways to produce a link between an STM tip and a substrate consist in either applying a voltage pulse locally melting the material [6] or in crashing the tip into the sample and then retracting it thereby pulling out a meniscus. This review will be focused on the 
similar break-junction technique where a lithographically prepared free-standing bridge is torn and thus thinned to atomic size diameter by bending the sample [7]. The AFM patterning method [3] has the drawback that the oxidation continues in an uncontrolled manner after turning off the voltage, although quantum steps in the conductance are nicely observed in exactly this time window. STM retraction curves show a similar stepwise decrease in multiples of the conductance quantum $G_{0}=2 e^{2} / h[6]$ whereas electrolyte contacts can be switched between no connection and one or several conductance quanta [5]. Mechanically controllable break-junctions (MCBs) make available a continuous observation of the transport properties from the bulk connection down to the single-atom constriction and even the tunnel regime, that is, they allow tuning of the contact transparency over the full range. The free suspension excludes influence of a substrate or surrounding medium, and the bridge geometry avoids principle asymmetries from the structure of the leads in contrast to the STM configuration with a flat substrate facing a sharp tip.

\section{Break junctions}

The realization of a MCB begins with a metallic stripe with a constriction of about $100 \mathrm{~nm}$ in width and length fabricated by electron-beam lithography, evaporation, and lift-off on top of an insulating polyimid layer on a bronze sub-

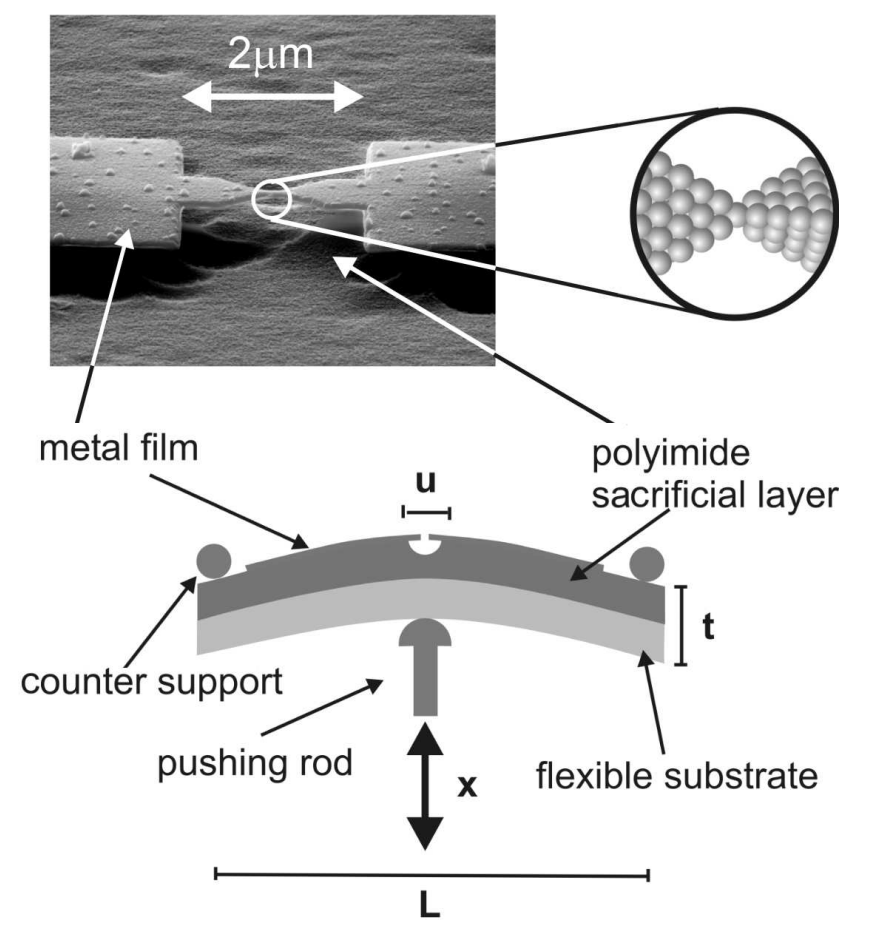

Fig. 1. Bottom: setup of sample in a bending mechanism. Top: electron microscope image of break-junction with a scheme (idealized) of atomic configuration at the contact. 
strate. A part of the polyimid is then removed in an oxygen plasma. The process changes the polyimid isotropically and thus the narrowest part of the metal becomes completely underetched (Fig. 1 top). The sample is then placed between two counter supports and a pushing rod (Fig. 1 bottom). The flexibility of bronze allows bending even at cryogenic temperatures. The last connection, before the narrow metallic bridge finally breaks off, will consist of a single atom. The contact ends will join together again on retracting the rod, and thus a MCB can be opened and closed many times. Mechanical control in the micrometer range of the position $x$ of the pushing rod is sufficient to vary the length $u$ of the bridge by a few angstrom because of the high displacement ratio $\mathrm{d} u / \mathrm{d} x=6 t u / L^{2} \sim 10^{-5}$, where $u$ is the length of the free-standing bridge of about $2 \mu \mathrm{m}, t$ is the thickness of the sample of a fraction of a millimeter and $L$ is the distance between the counter supports of about $1-1.5 \mathrm{~cm}$.

\section{Conduction quantization and channel ensemble}

For the MCBs, while bending the sample, a stepwise decrease in the conductance is observed. Individual opening curves as in Fig. 2a or the lower inset of Fig. 3 are not reproducible, because the exact atomic arrangement will differ each time. (The regions immediately to the left and right from the point contact need not at all be regular pyramids as drawn in Fig. 2a just to illustrate the neck becoming thinner.) Nevertheless, conductance histograms over many samples as well as many opening and closing cycles (Fig. 2b) reveal that the plateaus are preferably found at certain material specific values, these being, however, non-integer multiples of the conductance quantum. For example, for gold peaks occur at $0.9 G_{0}, 1.8 G_{0}, 2.9 G_{0}$, while their positions are $0.8 G_{0}, 1.9 G_{0}$, and $3.2 G_{0}$ for aluminum (see [1] for other materials). For the STM and electrolyte experiments cited the conductance jumps in multiples of $G_{0}$ when the diameter of the constriction is varied and a simple waveguide picture [9] seems to hold. The conductance values of MCBs also change discontinuously, but in general are no integer multi-
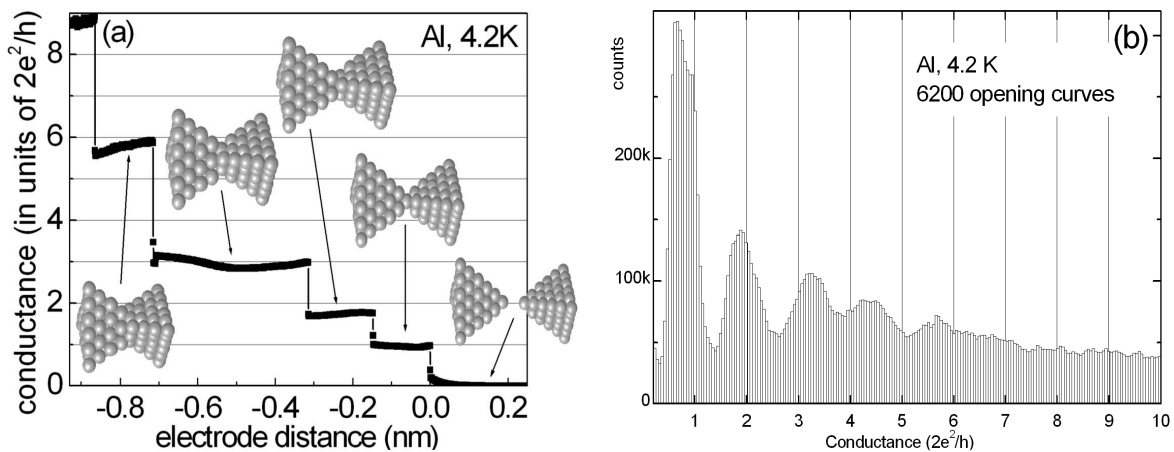

Fig. 2. (a) Opening curve of Al-MCB at $4.2 \mathrm{~K}$ (by courtesy of Elke Scheer). (b) Histogram of conductance values. Reprinted with permission from Ref. [8]. Copyright (1997) by the American Physical Society. 
ples of $G_{0}$. This is understood by assigning here each of the contributing transport channels $i$ to a transmission probability $\tau_{i}$ between zero and one [10]. The total conductance $G$ is therefore given by the Landauer formula:

$$
G=\frac{2 e^{2}}{h} \sum_{i} \tau_{i}, \quad 0<\tau_{i} \leq 1 .
$$

The number of channels often changes with the jumps in the opening curves which are attributed to sudden atomic reconfigurations, but it stays constant under elastic deformation corresponding to the conductance plateaus. Although only the total conductance is accessible to measurement, the channel ensemble $\left\{\tau_{i}\right\}$ can be obtained from the whole current-voltage characteristics $(I-V)$ in the superconducting state (Fig. 3) for any fixed contact position, that means at any single point on an opening curve. In the lower inset of Fig. 3 the horizontal bar indicates how to convert the time scale for bending the sample into a change in the length of the bridge, like it has been calibrated from the tunneling regime and given relative to the breaking point in Fig. 2a. Superconductivity is induced via the proximity effect for materials not becoming superconducting by themselves at low temperatures [11]. In Fig. 3 four $I-V$-curves are shown for four different positions of an aluminum-MCB reaching from a contact so large as to have at least one nearly perfectly transmitting channel to a fully opened bridge behaving like a tunnel junction. The applied voltage is indicated in units of the half-gap $\Delta$ of the superconductor and the current in each case is normalized to the normal-state

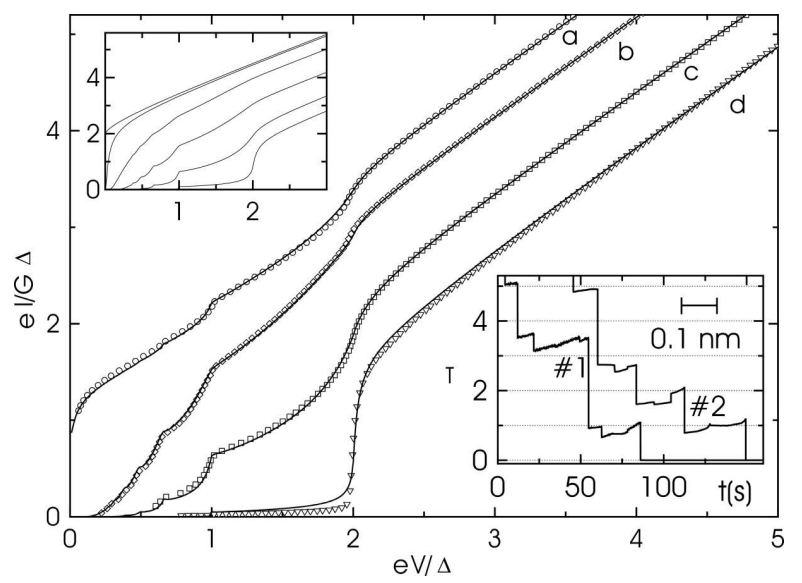

Fig. 3. Measured $I-V \mathrm{~s}$ and best fits at four opening positions of an aluminium break-junction at $30 \mathrm{mK}$. Total transmissions and channel ensembles $\left\{\tau_{i}\right\}$ are: (a) $G=1.747,\{0.997,0.46,0.29\}$, (b) $G=0.85,\{0.74,0.11\}$, (c) $G=0.88,\{0.46,0.35,0.07\}$, (d) $G=0.025,\{0.025\}$. Upper inset: calculated $I-V$ s for a single channel of transmission (from bottom to top) $\tau=0.1,0.4,0.7,0.9,0.99,1.0$. Lower inset: typical opening curves. Reprinted with permission from Ref. [7]. Copyright (1997) by the American Physical Society. 
conductance $G$ as well as units in multiples of $\Delta / e$. In the superconducting state, multiple Andreev reflections give rise to a subgap structure at $\mathrm{eV}$ below $2 \Delta$ with enough non-linearities to extract the number of channels as well as the $\tau_{i}$-values. This is done by fitting the $I-V$ with a sum of calculated single-channel curves, some examples of which are shown in the upper inset of Fig. 3; the axis units are the same as for the main diagram. The numbers of channels on the last plateaus of opening curves corresponding to single-atom contacts are linked to the chemical valence of the elements used [12]. Monovalent metals, like gold or silver, show one conduction channel, whereas $s p$-metals, like aluminum or lead, have three ones but five channels are found for transition metals such as niobium.

\section{Theoretical model}

For transport channels with transmission not small compared to one, especially in the superconducting state, multiple (Andreev) reflection processes become important. The different-order contributions, corresponding to the abrupt derivative changes $\mathrm{d} I / \mathrm{d} V$ in the subgap region and the excess currents at voltages $\mathrm{eV}>2 \Delta$ in Fig. 3, are not independent, though. The basis of a Green functions formalism [13] for calculation of $I-V \mathrm{~s}$ is a Dyson equation to establish a transfer rate-function self-consistently. The concept that any transfer is a single transfer or a single transfer followed by any transfer leads to

$$
T=\Sigma+\Sigma g T \Rightarrow T=(1-\Sigma g)^{-1} \Sigma=\Sigma+\Sigma g \Sigma+\Sigma g \Sigma g \Sigma+\ldots,
$$

where $T$ is the desired transfer matrix between wave-functions of the reservoirs on both sides of the contact, $\Sigma$ denotes the single-hopping amplitude, and $g$ describes the time evolution of the uncoupled reservoirs. The Dyson equation (2) implicitly contains interactions between the two contact sides to all orders. With the closed form solutions for the rate-amplitudes $T$ the resulting current is found from the excess of electron respectively hole transfer in one versus the other direction. This theory can be extended to a series of two junctions with channels of arbitrary transmission, where accumulation of charge on the middle island may hinder the transport. The interplay of such Coulomb blockade and multiple Andreev reflection is a subject of our current research.

\section{Outlook}

Break junctions due to their controllable contact distance in the subnanometer range also provide ideal pairs of electrodes to investigate conductance through freely suspended clusters or molecules. In analogy to the single-atom contacts, transport through molecules [14] can be understood in terms of conduction channels arising from molecular orbitals [15]. $\mathrm{C}_{60}$-fullerenes trapped between gold electrodes also have nonlinear $I-V \mathrm{~s}$ [16]. Also for DNA, jumps in the conductance due to different stages of stretching may be interesting. For $\mathrm{Si}_{4}$-clusters different kinds of non-linearities in the $I-V$-characteristics are predicted depending on the electrodes being of gold or aluminum. This again emphasizes that in this field 
of mesoscopic physics, transport properties do not only depend on the entity in the contact center, but also on the lead material, the shape of the whole contact region, and how the central atom or molecule is embedded in its surroundings.

\section{References}

[1] N. Agraï, A.L. Yeyati, J.M. van Ruitenbeek, Phys. Rep. 377, 81 (2003).

[2] F. Pérez-Willard, J.C. Cuevas, C. Sürgers, P. Pfundstein, J. Kopu, M. Eschrig, H.v. Löhneysen, Phys. Rev. B 69, 140502 (2004).

[3] E.S. Snow, D. Park, P.M. Campbell, Appl. Phys. Lett. 69, 269 (1996).

[4] C.Z. Li, N.J. Tao, Appl. Phys. Lett. 72, 894 (1998).

[5] F.-Q. Xie, L. Nittler, Ch. Obermair, Th. Schimmel, Phys. Rev. Lett. 93, 128303 (2004).

[6] J.I. Pascual, J. Méndez, G. Gómez-Herrero, A.M. Baró, N. García, V.T. Binh, Phys. Rev. Lett. 71, 1852 (1993).

[7] E. Scheer, P. Joyez, D. Esteve, C. Urbina, M.H. Devoret, Phys. Rev. Lett. 78, 3535 (1997).

[8] A.I. Yanson, J.M. van Ruitenbeek, Phys. Rev. Lett. 79, 2157 (1997).

[9] B.J. van Wees, H. van Houton, C.W.J. Beenakker, J.G. Williamson, L.P. Kouwenhoven, D. van der Marel, C.T. Foxon, Phys. Rev. Lett. 60, 848 (1988).

[10] J.C. Cuevas, A.L. Yeyati, A. Martin-Rodero, Phys. Rev. Lett. 80, 1066 (1998).

[11] E. Scheer, W. Belzig, Y. Naveh, M.H. Devoret, D. Esteve, C. Urbina, Phys. Rev. Lett. 86, 284 (2001).

[12] E. Scheer, N. Agraï, J.C. Cuevas, A.L. Yeyati, B. Ludoph, A. Martin-Rodero, G.R. Bollinger, J.M. van Ruitenbeek, C. Urbina, Nature 394, 154 (1998).

[13] J.C. Cuevas, A. Martin-Rodero, A.L. Yeyati, Phys. Rev. B 54, 7366 (1996).

[14] J. Reichert, R. Ochs, D. Beckmann, H.B. Weber, M. Mayor, H. v. Löhneysen, Phys. Rev. Lett. 88, 176804 (2002).

[15] J. Heurich, J.C. Cuevas, W. Wenzel, G. Schön, Phys. Rev. Lett. 88, 256803 (2002).

[16] T. Böhler, J. Grebing, A. Mayer-Gindner, H.v. Löhneysen, E. Scheer, Nanotechnology 15, 465 (2004). 